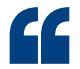

the kinase

portion of TRPM7 binds to components of chromatinremodelling complexes and phosphorylates histones

EPIGENETICS

\title{
The TRPM7 ion channel modifies histones
}

The cationic ion channel TRPM7 (transient receptor potential cation channel subfamily $\mathrm{M}$ member 7) has been referred to as a 'chanzyme', because it conducts $\mathrm{Zn}^{2+}, \mathrm{Mg}^{2+}$ and $\mathrm{Ca}^{2+}$ ions and harbours a Ser/Thr kinase domain at its carboxyl terminus. However, why these two functions are coupled in TRPM7, and how TRPM7 carries out a proposed role in regulating gene activity, is unclear. Krapivinsky et al. now show that, after release from the channel domain, the kinase portion of TRPM7 binds to components of chromatin-remodelling complexes and phosphorylates histones; this function is aided by a TRPM7-dependent increase in cytosolic $\mathrm{Zn}^{2+}$ levels.

While analysing anti-TRPM7 immunoprecipitation products, the authors observed a number of protein species with a lower molecular weight than full-length TRPM7, suggesting that TRPM7 is

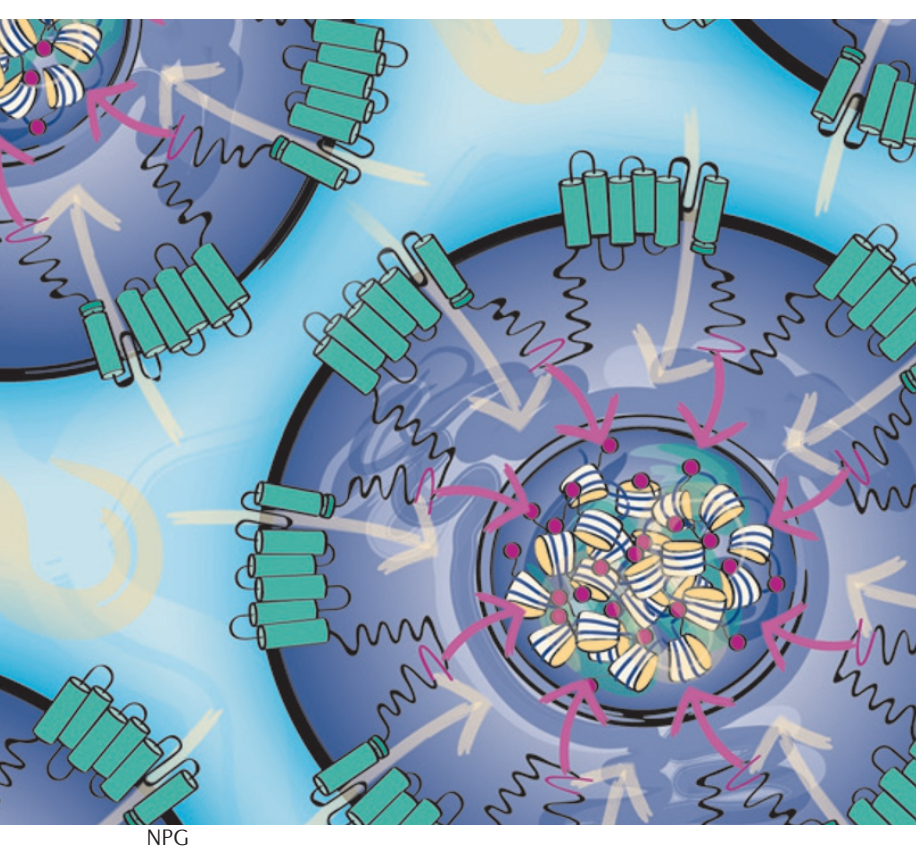

cleaved. They named these fragments TRPM7-cleaved kinases (M7CKs), as they all contained the $\mathrm{C}$ terminus of TRPM7. Of note, different cell types displayed specific TRPM7 cleavage patterns, which suggested that the cleaved fragments might have distinct functions.

The authors next screened for proteins that interact with the TRPM7 C terminus and, surprisingly, identified several nuclear proteins that potentially do so. As they had observed M7CKs in the nucleus, they pursued these possible interactions further. They found that, in mouse embryonic stem (ES) cells, TRPM7 interacted with several components of chromatin-remodelling complexes, such as the PRC1 (Polycomb repressor complex 1) components RYBP (RING1 and YY1-binding protein) and RNF2 (RING finger protein 2), the PRC2 component histone-Lys $\mathrm{N}$-methyltransferase EZH2 and the DNA helicase INO80 component YY1. The proteins that interacted with TRPM7 also associated with M7CKs and preferentially bound to specific M7CKs fragments, which perhaps explains why multiple M7CKs exist in different cell lines. Thus, M7CKs interact with various components of the PRC1, PRC2 and INO80 complexes.

How do M7CKs get into the nucleus? The $\mathrm{C}$ terminus of TRPM7 contains a putative nuclear localization signal, which may facilitate the nuclear entry of some M7CKs. The authors also found that co-expression of M7CKs with the nuclear proteins RYBP or RNF2 increased the levels of nuclear M7CKs. RYBP-M7CK interactions were blocked by the addition of a heavy metal chelator, which could be rescued by the addition of $\mathrm{Zn}^{2+}$. Furthermore, binding of proteins containing a zinc-finger domain to M7CK was $\mathrm{Zn}^{2+}$-dependent. As the cytosolic concentration of $\mathrm{Zn}^{2+}$ was lower in TRPM7-null ES cells than in wild-type ES cells, TRPM7 channel activity might increase the concentration of $\mathrm{Zn}^{2+}$ in the cytosol to facilitate M7CK-chromatin remodelling complex interactions.

So, what do M7CKs phosphorylate in the nucleus? The authors observed that, globally, the phosphorylation of histone $\mathrm{H} 3$ at Ser10, Ser28 and Thr3 was suppressed in TRPM7-null ES cells. The acetylation of histone $\mathrm{H} 3$ at Lys9 and Lys27 was also reduced in TRPM7-null ES cells, which is in agreement with the previous finding that these acetylation events depend on phosphorylation at the adjacent Ser residues. Reconstitution of this ES cell line with a wild-type M7CK, but not with a kinase-dead M7CK, rescued the suppression of histone $\mathrm{H} 3$ phosphorylation. Various genes were downregulated in TRPM7-null ES cells, and this downregulation correlated with decreased phosphorylation of histone $\mathrm{H} 3$ at Ser10 in the promoters of the downregulated genes; both events were restored upon expression of a M7CK.

Thus, this study suggests that a TRPM7-dependent increase in cytosolic $\mathrm{Zn}^{2+}$ levels promotes the association of the cleaved TRPM7 kinase portion with certain chromatin-remodelling factors, which enables M7CKs to promote histone phosphorylation in the nucleus and thereby regulate gene expression. Katharine H. Wrighton

ORIGINAL RESEARCH PAPER Krapivinsky. G. et al. The TRPM7 chanzyme is cleaved to release a chromatin-modifying kinase. Cell, 157, 1061-1072 (2014) 